\title{
CMOS Active Bandpass Filter Using Compacted Synthetic Quasi-TEM Lines at $C$-Band
}

\author{
Ching-Kuang C. Tzuang, Fellow, IEEE, Hsien-Hung Wu, Student Member, IEEE, Hsien-Shun Wu, Member, IEEE, \\ and Johnsea Chen
}

\begin{abstract}
This paper presents a fully monolithic transmission-line-based active bandpass filter (BPF) fabricated in a 0.18- $\mu \mathrm{m}$ standard complementary metal-oxide-semiconductor (CMOS) technology. The half-wavelength resonators are realized by synthetic quasi-TEM complementary conducting-strip transmission lines (CCS TLS). To lower the insertion loss of the BPF, the differential nMOS cross-coupled pairs are combined with the parallel resonators. Besides, the active devices and CCS TLS are vertically integrated on the standard CMOS substrate. The $Q$-enhanced resonator, which is comprised of a CCS TL and an nMOS cross-coupled pair, is theoretically investigated. Simulation results indicate that the $Q$ factor of the resonator can be increased from 3.4 to 84.0 at $6.53 \mathrm{GHz}$. Additionally, the prototype of the second-order BPF occupies an area of $1230 \mu \mathrm{m} \times 880 \mu \mathrm{m}$, and the measured results demonstrate that the center frequency is 6.02 GHz with a bandwidth of $1.14 \mathrm{GHz}$. The $P_{1 \mathrm{~dB}}$ and insertion loss are $-15.2 \mathrm{dBm}$ and $2.2 \mathrm{~dB}$, respectively, when the $\mathrm{BPF}$ consumes $3.0 \mathrm{~mA}$ from a 1.8-V supply. A two-port noisy network is also reported to examine the noise figure (NF) of the proposed BPF. Theoretical results reveal that the NF is $11.38 \mathrm{~dB}$ at $6.0 \mathrm{GHz}$, with a difference of less than $7.2 \%$ among the measured data.
\end{abstract}

Index Terms-Active bandpass filter (BPF), $C$-band, CMOS, transmission line (TL).

\section{INTRODUCTION}

$\mathbf{O}$ N-CHIP radio or RF systems-on-chip (RF SOCs) incorporating monolithic complementary metal-oxide-semiconductor (CMOS) bandpass filters (BPFs) have become increasingly attractive in the world of a congested frequency spectrum for personal communications service (PCS) band [1], wideband code-division multiple-access (WCDMA) receivers [2], and time-division-duplex (TDD) systems [3]. Elimination of off-chip filters often means adding RF performance, improving selectivity requirements, reducing noise pick-up, and lowering overall RF power consumption [2], [3]. On-chip CMOS passive resonators formed of transmission lines (TLs) typically have quality factors ( $Q$ factors) proportional to the square root of the operating frequency. A carefully designed CMOS BPF at millimeter-wave frequency generally provides an adequate $Q$ factor. Therefore, a passive BPF at 30 and

Manuscript received April 8, 2006; revised May 30, 2006.

C.-K. C. Tzuang and H.-S. Wu are with the Graduate Institute of Communication Engineering, Department of Electrical Engineering, National Taiwan University, Taipei, Taiwan 106, R.O.C. (e-mail: cktzuang@ cc.ee.ntu.edu.tw).

H.-H. Wu is with the Department of Communication Engineering, National Chiao Tung University, Hsinchu, Taiwan 300, R.O.C. (e-mail: hhwu_msn@yahoo.com.tw).

J. Chen is with the Cadence Methodology Service Company, Taipei, Taiwan 116, R.O.C.

Digital Object Identifier 10.1109/TMTT.2006.881507
$40 \mathrm{GHz}$ have been found to perform reasonably well [4], [5]. However, most wireless applications operate below $C$-band (4-8 GHz) where the CMOS on-chip spiral inductor necessary for carrying out the resonator design has a fairly low $Q$ factor, of typically 5 or below [6, Table I]. This paper presents the recent advance in the state-of-the-art CMOS active BPF design achieving a small size, low passband loss, high outband rejection, low power consumption, low noise figure (NF), and high input 1-dB compression point. The literature survey indicates that most active CMOS BPFs below the $C$-band incorporate inductors for resonator designs, e.g., actively $Q$-enhanced coupled inductors [2], [7], emulated coupled inductors [6], energy-recovered spiral inductors [8], and $Q$-enhanced $L C$ bandpass biquads [9].

This paper presents a novel approach based on a microwave filter design procedure incorporating low- $Q$ half-wavelength resonators loaded by active circuits to compensate losses [10]. In contrast to the recently reported $C$-band passive lumped-element filter [11], which was fabricated in a highly resistive silicon substrate with a resistivity 100 times that of a typical CMOS foundry wafer, the proposed design methodology adopts synthetic quasi-TEM TLs on a standard CMOS substrate, rendering a high-performance miniaturized active BPF design. The previously published research briefly reported the measurement results of the proposed active BPF [10]. However, this paper explores in detail the design of the active TL resonator based on a complementary conducting-strip transmission line (CCS TL) followed by the differential- and common-mode analyses on the active resonator in Section II. Section III then presents a practical example with the design parameters and experimental characterizations. Section IV then investigates the NF of the presented active BPF with a two-port noisy network. Conclusions are finally drawn in Section V.

\section{CMOS TL-BASED RESONATORS}

\section{A. $\operatorname{CMOS~CCSTL}$}

Recently the synthetic quasi-TEM TL so-called CCS TL had experimentally demonstrated its application in [10], [12], [13]. The CCS TL is made of a unit cell, which has dimensions much smaller than the operating wavelength, typically one-thousandth. The unit cell consists of a mesh ground and a four-arm signal trace. The guiding characteristics of the CCS TL had been investigated intensively, showing the following features [12]-[14]. First, the characteristic impedance and propagation constant of the CCS TL can be controlled by the varying geometric parameters such as the width of the signal 


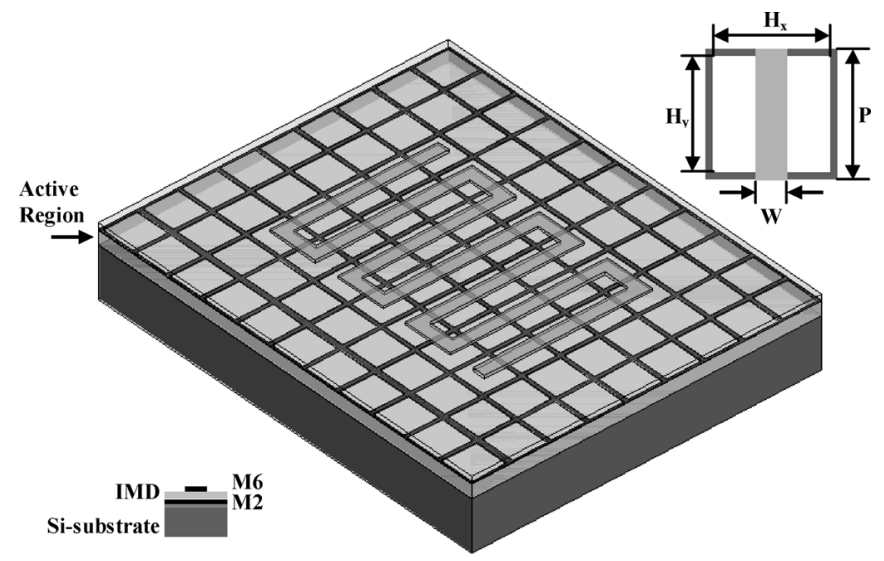

Fig. 1. Monolithic CCS TL of $7 \times 5$ unit cells integrating above the active devices.

trace and the mesh area of the mesh ground plane. Second, the real estate of the CCS TL-based passive circuit is proportional to the period of unit cell, which is scalable with advance in photolithography. Third, the mesh ground plane can provide good electromagnetic (EM) shields to avoid cross coupling of circuits integrated by the CCS TL. Fig. 1 reports for the first time that the CCS TL is right on top of transistor region. The inset of Fig. 1 shows the cross-sectional view of the CCS TL adopted in this paper, in which top metal forms a meandered signal path. M2 is applied to realize the mesh ground plane, which is sandwiched between the CCS TL and the first metal layer (M1) for interconnections between active devices. Such connections also include the biasing paths for the terminals (source, drain, and gate) in a MOS transistor backbone buses for paralleling the MOS transistors.

The guiding characteristics of the CCS TL on the silicon substrate shown in Fig. 1 were investigated by performing full-wave EM simulations using Ansoft's finite-element-based High-Frequency Structure Simulator (HFSS). The following material and structural parameters, which were defined in the EM simulations, were specified based on a typical $0.18-\mu \mathrm{m}$ CMOS 1 P6M process. The thicknesses of M6 and M2 metal layers are 2.0 and $0.55 \mu \mathrm{m}$, respectively. The relative dielectric constant and the thickness of inter-media-dielectric (IMD) sandwiched between M6 and M2 are 4.0 and $5.88 \mu \mathrm{m}$, respectively. The thickness and conductivity of the silicon substrate are $482.6 \mu \mathrm{m}$ and $11 \mathrm{~S} / \mathrm{m}$, respectively. The linewidth (W) of the CCS TL is $30.0 \mu \mathrm{m}$, the period $(\mathrm{P})$ of the unit cell is $44 \mu \mathrm{m}$ and the mesh area $\left(H_{x} \times H_{y}\right)$ is $40 \mu \mathrm{m} \times 40 \mu \mathrm{m}$.

The TL parameters, including complex characteristic impedances and propagation constants, were extracted from the twoport scattering analyses [12]. Fig. 2 shows the extracted results of the typical CCS TL design example of Fig. 1 based on the above-mentioned parameters.

From 1.0 to $8.0 \mathrm{GHz}$, the real part of the characteristics impedance $\left(Z_{c}\right)$, which is the solid line plotted in Fig. 2(a), nearly keeps at a constant value of $34.2 \Omega$. The imaginary part of $Z_{c}$ is capacitive, ranging from -11.7 to $-2.04 \Omega$. The normalized phase constant shown in solid line in Fig. 2(b) illustrates the value of 1.86 at the desired operating frequency.

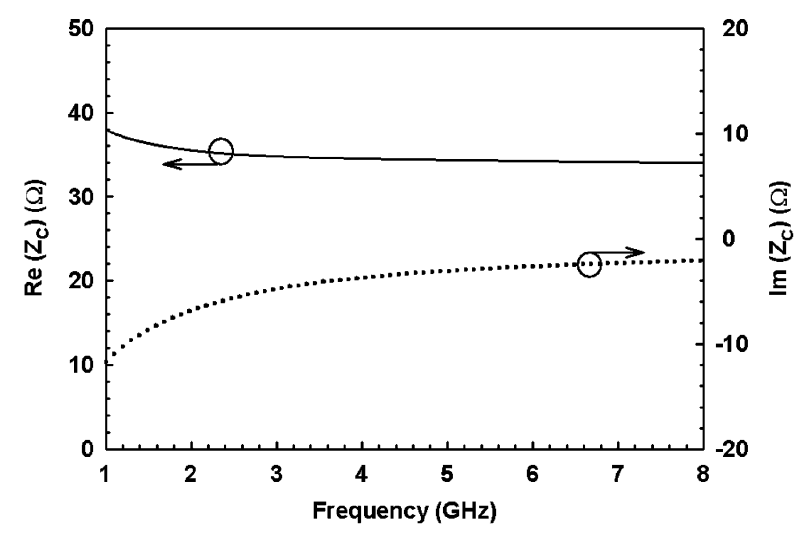

(a)

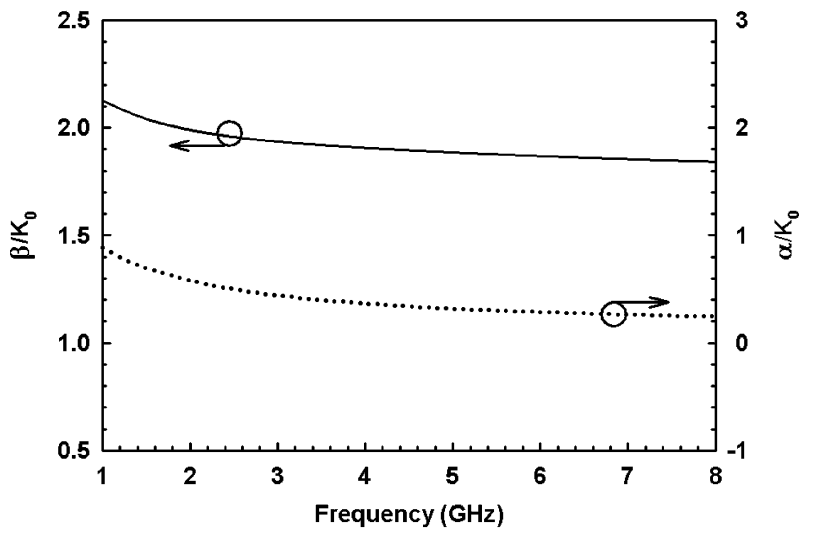

(b)

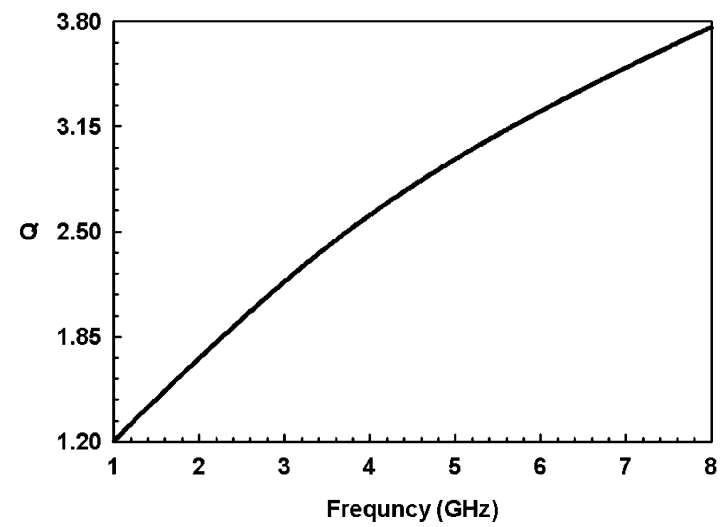

(c)

Fig. 2. Guiding characteristics of the meandered compacted CMOS-based CCS TL of Fig. 1. (a) Complex characteristic impedance. (b) Normalized complex propagation constant. (c) $Q$ factor.

Therefore, we can estimate the physical length of a half-wavelength CCS TL at $6.0 \mathrm{GHz}$ to be $13210-\mu \mathrm{m}$ long and such TL can be compacted in the area of $792 \mu \mathrm{m} \times 792 \mu \mathrm{m}$ by using the meandered CCS unit cells with a period of $44.0 \mu \mathrm{m}$.

The normalized attenuation constant, which is plotted by the dotted symbol in Fig. 2(b), however, shows a relatively high loss aspect of the TL. In the low frequency limit $(1 \mathrm{GHz})$, the metal thickness employed in the CCS TL is smaller than the skin depth, thus we observe larger attenuation losses. Fig. 2(c) plots the $Q$ factor of the CCS TL against frequency, showing $2.19,2.94$, and 3.40 at $3.0,5.0$, and $6.53 \mathrm{GHz}$, respectively. The $Q$ factors employed in our design of an active resonator 


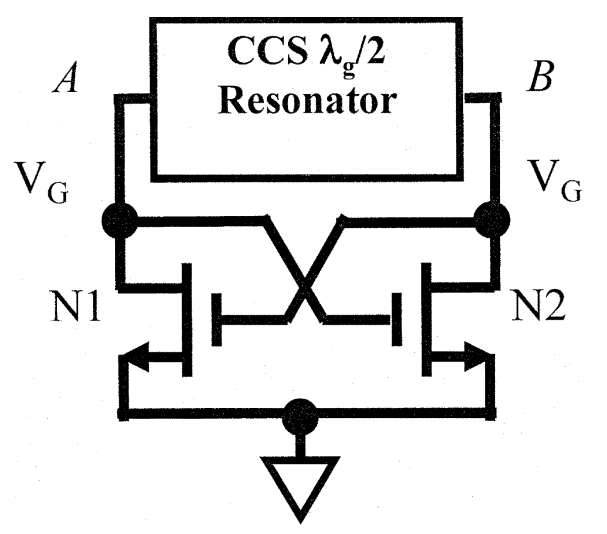

Fig. 3. $Q$-enhanced CCS half-wavelength resonator incorporating an nMOS cross-coupled pair.

are comparable, but smaller than those of inductor-based design [1]-[3], [6]-[9].

Since the $Q$ factor of the CCS TL suffers from the process limitation, the losses of CCS-TL-based resonators need to be compensated. In Section II-B, a MOS-based active network is reported for elevating the $Q$ factor of the CCS half-wavelength resonator.

\section{B. Q-Enhanced Monolithic Half-Wavelength Resonator}

Fig. 3 shows the concept of a $Q$-enhanced complementary conducting-strip (CCS) half-wavelength resonator. A cross-coupled pair, which consists of two identical nMOS transistors, is integrated into a half-wavelength resonator. The drain terminal of N1 is directly connected to the gate terminal of $\mathrm{N} 2$ and vise versa. Two transistors are biased at the same dc potential $\left(V_{G}\right)$, and the drain terminals of both $\mathrm{N} 1$ and $\mathrm{N} 2$ are directly loaded with a CCS half-wavelength resonator forming a new composite resonator.

Since the active resonator will be excited single endedly, not differentially, both common- and differential-mode signals will exit in the active resonator structure. Fig. 4(a) and (b) illustrates the equivalent circuits for differential- and common-mode excitations, respectively.

When a differential-mode signal transmits into a cross-coupled nMOS transistors pair and establishes a positive feedback, a virtual ground is formed at the symmetric plane rendering a negative differential resistance $-R_{d}$ in Fig. 4(a) with magnitude approximately equal to the inverse of transconductance of the cross-coupled pair [15]. On the other hand, the capacitance across the resonator is approximately half of the combined capacitance $\left(C_{\mathrm{gs}}+C_{\mathrm{gd}}\right)$.

Since the potentials on the drain and gate terminals of the nMOS are equal under a common-mode excitation, the nMOS acts as a gate-drain-connected diode. Therefore, two parallel $R C$ networks are loaded with both sides of the half-wavelength resonator. The shunt resistance $R_{c}$ shown in Fig. 4(b) represents the small-signal resistive loss of the transistor operated in the saturation region. To make proper operation of the active BPF, the differential mode must prevail over the common mode in the passband. Since the cross-coupled pair can amplify the differential-mode signal and attenuate the common-mode signal, such a circuit characteristic can increase the common-mode rejection
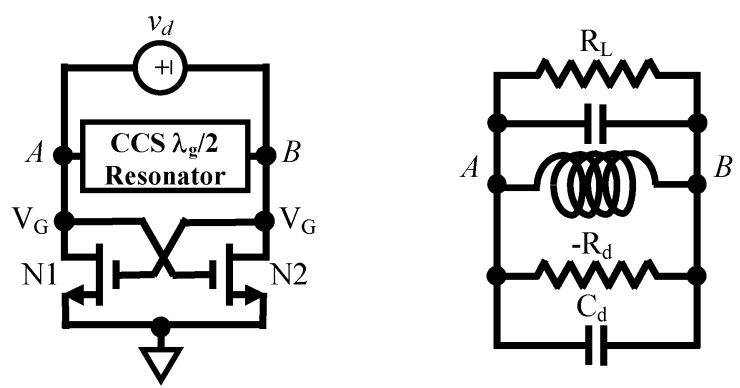

(a)
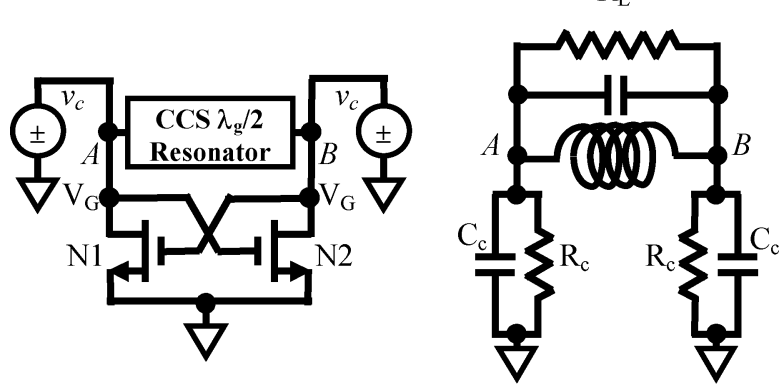

(b)

Fig. 4. Small-signal analyses of the $Q$-enhanced half-wavelength resonator. (a) Differential-mode analysis. (b) Common-mode analysis.

of the proposed $Q$-enhanced resonator, and relax the issue on symmetrical layout of the resonator during the filter integration.

The complex input impedance under differential-mode excitation was theoretically investigated by using Agilent's ADS2004A software. Through the analysis, the length and width of the two transistors were set at 0.18 and $80 \mu \mathrm{m}$, respectively. $V_{G}$ was isolated from the differential RF signal by an RF choke. The results illustrate that the value of $C_{d}$ and $-R_{d}$ were nearly constant from 1.0 to $8.0 \mathrm{GHz}$, revealing a broadband characteristic of the equivalent active $R C$ circuit. The total equivalent resistance ( $R_{\text {equ }}$ ) of the differentially driven active resonator can be expressed by

$$
R_{\mathrm{equ}}=\frac{-R_{d} \cdot R_{L}}{R_{L}-R_{d}}
$$

where $R_{L}$ represents the loss of the CCS half-wavelength resonator. Since the value of the frequency-dependent $R_{L}$ increases with increasing frequency, the active resonator tends to become more stable at frequency higher than the resonant frequency. Furthermore, the value of $-R_{d}$ is inversely proportional to the drain current of the nMOS transistors [15]. Thus, as shown in Fig. 5, $V_{G}$ can be applied to adjust proper negative resistance for realizing a stable half-wavelength resonator.

The inset in Fig. 6 depicts the schematic for extracting the unloaded $Q$ factor of the active half-wavelength resonator shown in Fig. 3. Two tiny capacitors of $0.01 \mathrm{fF}$ formed the EM coupling between the resonator and loads. Clearly the excitation was single ended. The size of the nMOS transistor was the same as that reported in Fig. 5, and the half-wavelength resonator was realized by using the meandered CCS TL reported in Section II-A. The value of $R_{L}$ of the CCS half-wavelength resonator and $-R_{d}$ of the cross-coupled pair 


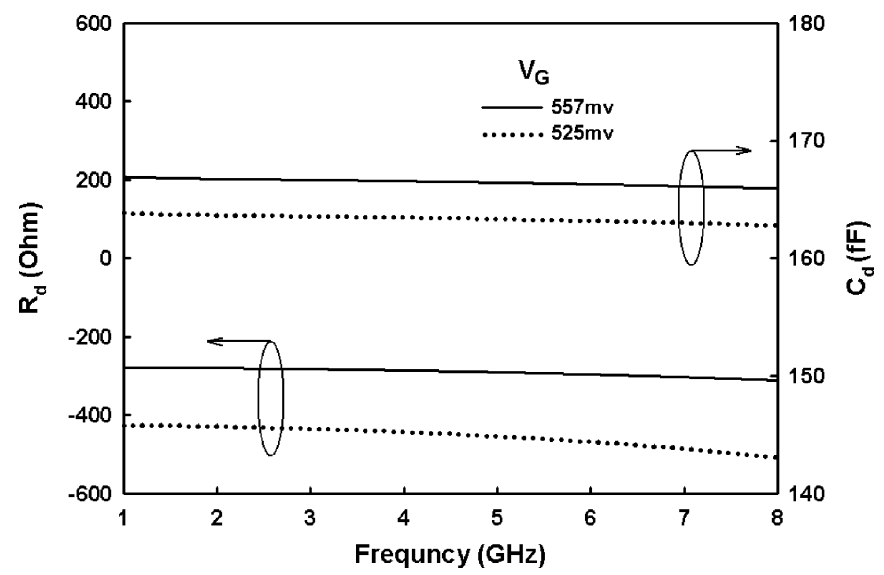

Fig. 5. Differential input impedance of a $0.18-\mu \mathrm{m}$ nMOS cross-coupled pair with length of $0.18 \mu \mathrm{m}$ and width of $80.0 \mu \mathrm{m}$.

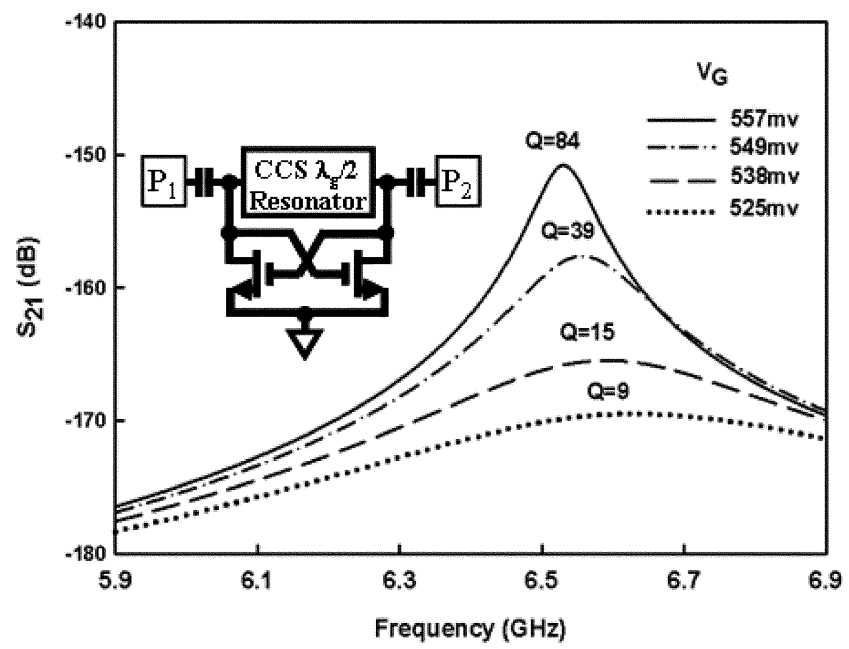

Fig. 6. Unloaded $Q$ factor of $Q$-enhanced half-wavelength resonator incorporating a $0.18-\mu \mathrm{m}$ nMOS cross-coupled pair.

biased at $557 \mathrm{mV}$ are 298.4 and $-301.34 \Omega$ at $6.53 \mathrm{GHz}$, respectively. Therefore, according to (1), Fig. 6 illustrates a stable active half-wavelength resonator.

The extracted $Q$ factors shown in Fig. 6 follow the definition of the unloaded $Q$ factor in [16]. Moreover, the magnitudes of the transducer gain of the weakly coupled active resonator are also illustrated in Fig. 6. The $Q$ factor was only 3.40 for the passive CCS half-wavelength resonator. With the active $Q$-enhanced circuit biased at $525,538,549$, and $557 \mathrm{mV}$, the enhanced $Q$ factors were $9,15,39$, and 84 , respectively. Notably, the resonant frequency of the $Q$-enhanced resonator was slightly shifted from 6.633 to $6.531 \mathrm{GHz}$ when $V_{G}$ was increased. Such frequency drift was caused by the increase of $C_{d}$ shown in Fig. 5 .

\section{CMOS TL-BASED ACTIVE BPF}

Fig. 7 shows the complete schematic of a second-order BPF incorporating the $Q$-enhanced half-wavelength resonators [10]. The $J$-inverters were realized by series capacitors $C_{1}, C_{2}$, and $C_{3}$. The design procedure of the BPF is well documented in [16]. In this practical example, $f_{c}$ of the BPF was located at $6.02 \mathrm{GHz}$, and bandwidth (BW) was $1.0 \mathrm{GHz}$ with a ripple of

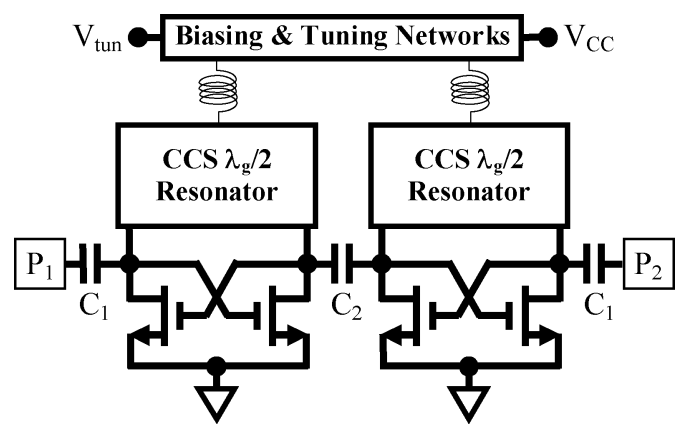

Fig. 7. Second-order CMOS TL-based BPF.

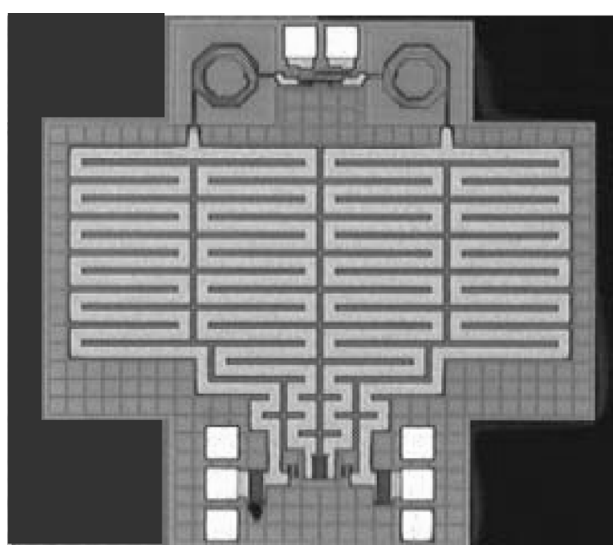

Fig. 8. Chip photograph of the prototype BPF in Fig. 7.

$0.2 \mathrm{~dB}$. The order of the BPF was two and the reference impedances of two terminals $\left(P_{1}\right.$ and $\left.P_{2}\right)$ were $50 \Omega$. The biasing and tuning networks controlled by $V_{\text {tun }}$ provided biasing currents for the nMOS cross-coupled pairs. These networks were isolated from the CCS TL resonators by the on-chip spiral inductors, as shown at the top of Fig. 8. Fig. 8 also illustrates the chip photograph of the prototype filter in Fig. 7.

The entire active BPF, including the CCS TLs, capacitors, inductors, active networks, and pads were fully integrated in a chip area of $1230 \mu \mathrm{m} \times 880 \mu \mathrm{m}$. The capacitor was realized with the so-called interdigital metal-oxide-metal (MoM) capacitors of top-three metal layers. In the realizations, $C_{1}$ was $380 \mathrm{fF}$ with an area of $45.9 \mu \mathrm{m} \times 79.8 \mu \mathrm{m}$, and $C_{2}$ was $220 \mathrm{fF}$ with an area of $41.9 \mu \mathrm{m} \times 52.8 \mu \mathrm{m}$, respectively. Additionally, the inductance of the on-chip spiral inductors was approximately $3.0 \mathrm{nH}$ and occupied an area of $251 \mu \mathrm{m} \times 247 \mu \mathrm{m}$.

The small-signal experiments were performed after the on-wafer short-open-load-thru (SOLT) procedures had been conducted by the vector network analyzer (VNA) Agilent E5091A. In the measurements, the prototype shown in Fig. 7 was biased by a supplying voltage $\left(V_{C C}\right)$ of $1.8 \mathrm{~V}$ with a current consumption of $3.0 \mathrm{~mA}$. The value of $V_{\mathrm{tun}}$ and the power level of input signals were set at $1.0 \mathrm{~V}$ and $-20 \mathrm{dBm}$, respectively. Additionally, the measured result was compared with simulations performed by Agilent's ADS2004A. Before the simulation, all the passive components including capacitors, inductors, and CCS TL were characterized by Ansoft's HFSS. The BSIM3 V3.2.4-based RF models used for active devices were provided by the foundry. Fig. 9(a) shows the comparisons 


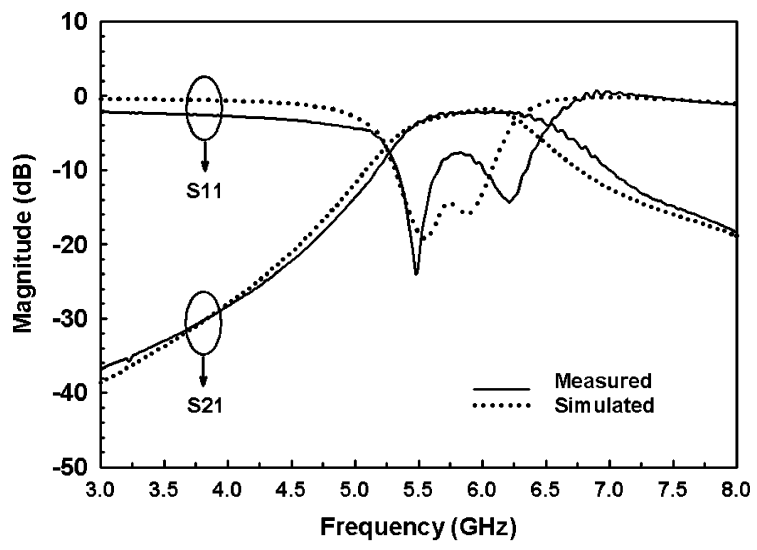

(a)

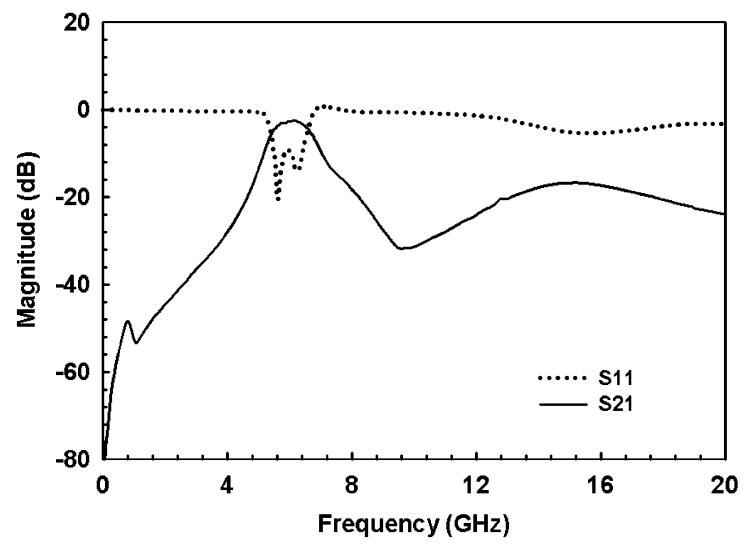

(b)

Fig. 9. Transmission and reflection characteristics of the active BPF in Fig. 8. (a) Comparison of measured and simulated data over $5-\mathrm{GHz}$ narrow $\mathrm{BW}$. (b) Measured responses across $20-\mathrm{GHz}$ broad BW for investigating spurious responses.

from 3.0 to $8.0 \mathrm{GHz}$, revealing good agreement between the simulated and measured data, except for the return loss at passband. The slight mismatch shows that the capacitive coupling between the two $Q$-enhanced half-wavelength resonators were well controlled through the $J$-inverter $C_{2}$, and parasitic coupling through the lossy substrate was not serious owing to the good EM shield from the meshed ground plane of the CCS TL. In other words, the CCS TL can effectively confine the EM propagations and eliminate the unwanted coupling of the adjacent signal lines in the compact layout.

The measured results of two-port scattering parameters based on the $50-\Omega$ system lead the following observations. The center frequency of the second-order $\mathrm{BPF}$ is $6.02 \mathrm{GHz}$, and the insertion loss is approximately $2.2 \mathrm{~dB}$ from 5.38 to $6.65 \mathrm{GHz}$. The $\mathrm{BW}$ is approximately $1.14 \mathrm{GHz}(5.26-6.40 \mathrm{GHz})$ with a return loss of $7.64 \mathrm{~dB}$. Two reflection zeros are identified at 5.47 and $6.20 \mathrm{GHz}$. Additionally, the prototype can reject the low-side signal approximately $28.18 \mathrm{~dB}$ at $4.0 \mathrm{GHz}$ and the high-side signal approximately $18.33 \mathrm{~dB}$ at $8.0 \mathrm{GHz}$. The spurious response of the prototype, which is observed in Fig. 9(b), is suppressed approximately $16.67 \mathrm{~dB}$ at $15.25 \mathrm{GHz}$.

The nonlinear characteristics of the prototype had also been investigated by measuring the input third-order intermodulation intercept point $\left(\mathrm{IIP}_{3}\right)$ and the $1-\mathrm{dB}$ compression point $\left(P_{1 \mathrm{~dB}}\right)$. For the measurement of $P_{1 \mathrm{~dB}}$, the signal generator Agilent

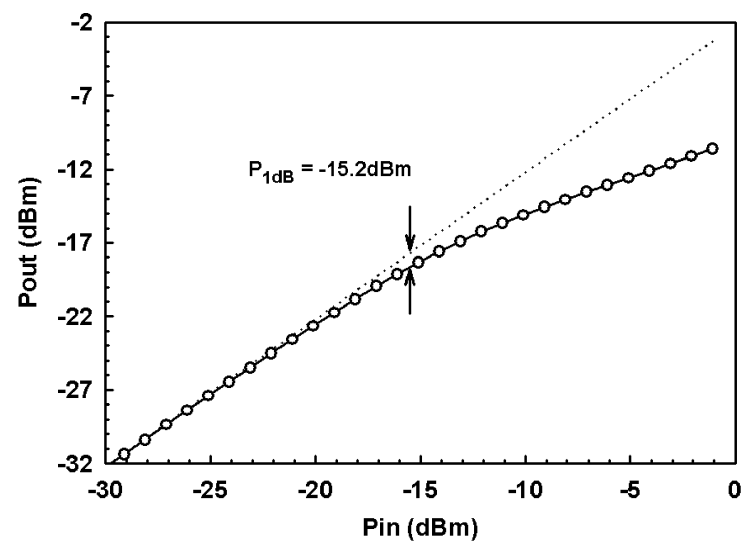

(a)

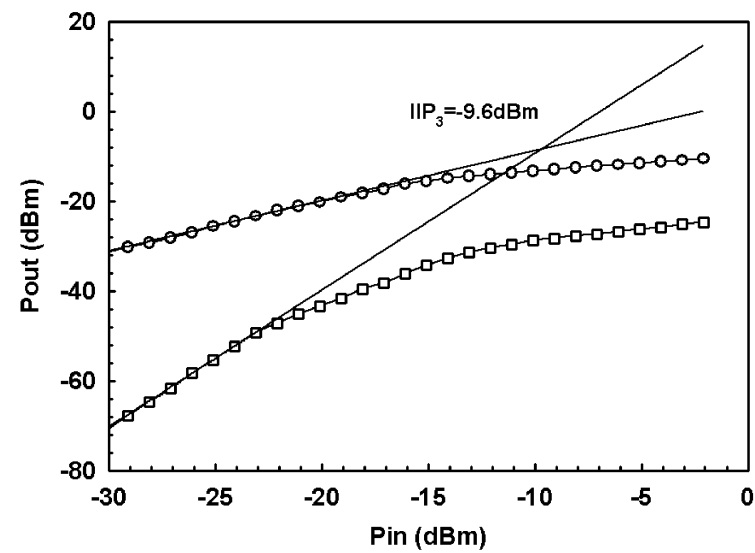

(b)

Fig. 10. Nonlinear characteristics of the active BPF in Fig. 8. (a) Input 1-dB compression point $\left(P_{1 \mathrm{~dB}}\right)$. (b) Input third-order intermodulation intercept point $\left(\operatorname{IIP}_{3}\right)$.

E8267D provided an input continuous wave $(\mathrm{CW})$ at $6.02 \mathrm{GHz}$, and the spectrum analyzer Agilent E4440A was applied to observe the output signals of the prototype. For the measurement of $\mathrm{IIP}_{3}$, two signal generators were applied to generate two fundamental frequencies centered at $5.8 \mathrm{GHz}$ with a separation of $10 \mathrm{MHz}$. The testing system, which includes the connectors and cables, were calibrated before the experiments. Additionally, the biasing conditions of the prototype were kept the same as those in the $S$-parameter experiments. The measured results, as shown in Fig. 10, indicate the input power levels for $P_{1 \mathrm{~dB}}$ and $\mathrm{IIP}_{3}$ are $-15.2 \mathrm{dBm}$ and $-9.6 \mathrm{dBm}$, respectively.

\section{NOISE ANALYSES OF TL-BASED ACTIVE BPF}

Experimental results in Fig. 9(a) indicate that the prototype is a passive filter with an insertion loss of $2.2 \mathrm{~dB}$ in the passband. According to the textbook definition, the NF of a passive two-port network is equivalent to the inverse of its available power gain [17]. However, the proposed BPF, as depicted in Fig. 7, is comprised of a CCS TL and differential nMOS cross-coupled pairs. The cross-coupled pairs not only provide negative resistance to elevate the $Q$ factor of the resonators, but also produce the noise simultaneously. Therefore, the noise contributions from the transistors need to be incorporated into the NF of the proposed BPF. Therefore, a noisy network is presented here to investigate the NF of the proposed BPF shown 


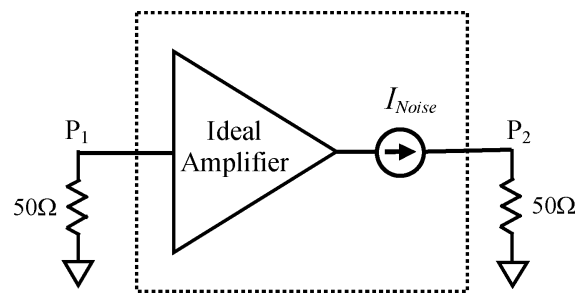

Fig. 11. Equivalent noisy two-port network of the prototype BPF in Fig. 7.

in Fig. 7. Fig. 11 illustrates the two-port network consists of an ideal amplifier and a noise current source. The gain of the ideal amplifier indicates the transmission coefficient of the BPF, and the noise current source at the output of the ideal amplifier represents the total noise current generated by the nMOS cross-coupled pairs.

The noise characteristics of an nMOS cross-coupled pair had been well documented in [18] and [19]. The differential output noise current spectral density of an nMOS cross-coupled pair is equivalent to the summation of thermal noise generated in the channels of two nMOS transistors [19]. Furthermore, the channel noise of an nMOS transistor operating in saturation can be quantified by an equivalent noise current between the drain and source terminals

$$
\bar{i}_{n}^{2}=4 k T \gamma g_{m}
$$

where $g_{m}$ and $\gamma$ are the transconductance and channel noise coefficient of an nMOS transistor, respectively [20], [21].

To demonstrate the feasibility of the proposed noisy network, the theoretical analyses were conducted with the two-port network illustrated in Fig. 11 and the transistor parameters reported in Section II-B. The value of $g_{m}$ derived according to the definition in [21] was $8.872 \mathrm{mS}$. The $\gamma$ value extracted from the small-signal noise analysis of the nMOS transistor was 1.012 at $6.0 \mathrm{GHz}$ [21]. Thus, the total noise current spectrum density of the nMOS cross-couple pairs was $6.436 e-22 \mathrm{~A}^{2} / \mathrm{Hz}$ at $298.15 \mathrm{~K}$. The calculated NF of the proposed BPF after following the procedures described in [22] was $11.38 \mathrm{~dB}$. Additionally, the calculation results from 5.5 to $6.0 \mathrm{GHz}$ were compared with those of the simulations and experiments, as illustrated in Fig. 12, revealing a difference of less than $7.2 \%$ on the noise analyses. The measurements, which were undertaken using the Agilent NF analyzer N8974A, reveal that the NF of the prototype was approximately $12.36 \mathrm{~dB}$ at $5.5 \mathrm{GHz}$, which was slight decreased to $10.92 \mathrm{~dB}$ at $5.8 \mathrm{GHz}$. Simulation results indicate that the NF of the proposed bandpass filer was 12.30 and $11.40 \mathrm{~dB}$ at 5.5 and $6.0 \mathrm{GHz}$, respectively. These good agreements indicate that the proposed noisy network is valid for predicting the NF of the BPF shown in Fig. 7.

Furthermore, the NF of the proposed BPF with different transistor width was also theoretically analyzed by following the same above-mentioned analytical procedures. Through the analyses, the characteristics of the BPF, including the insertion loss, reflection coefficient, and BW, were identical to those reported in Fig. 9(a).

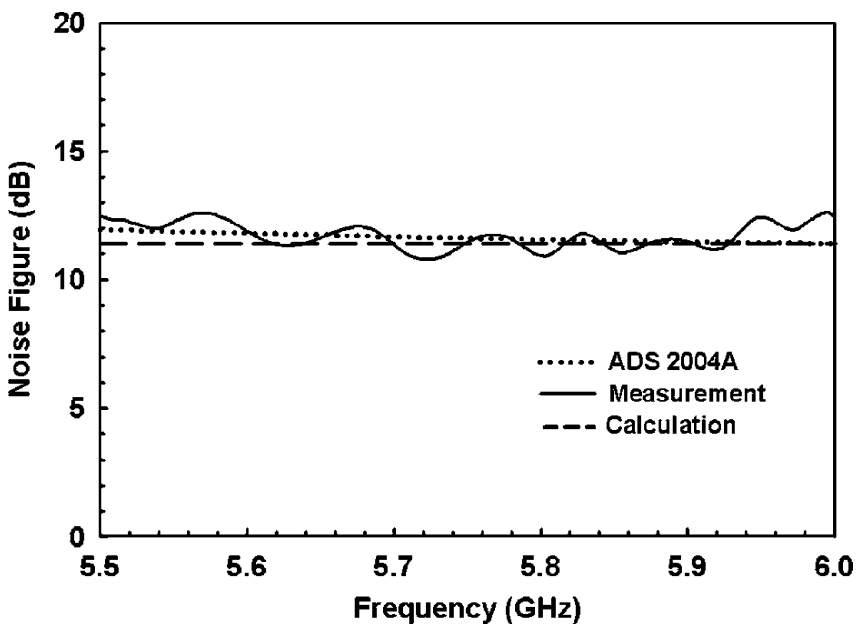

Fig. 12. NFs of the proposed BPF.

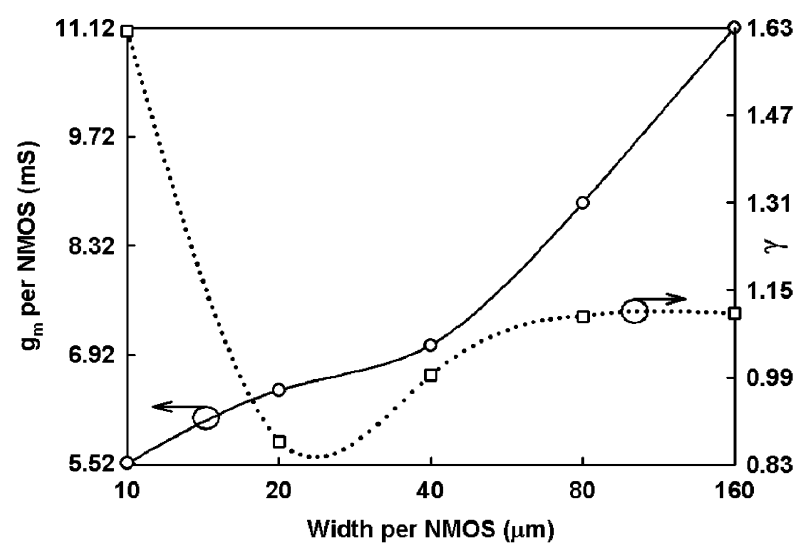

(a)

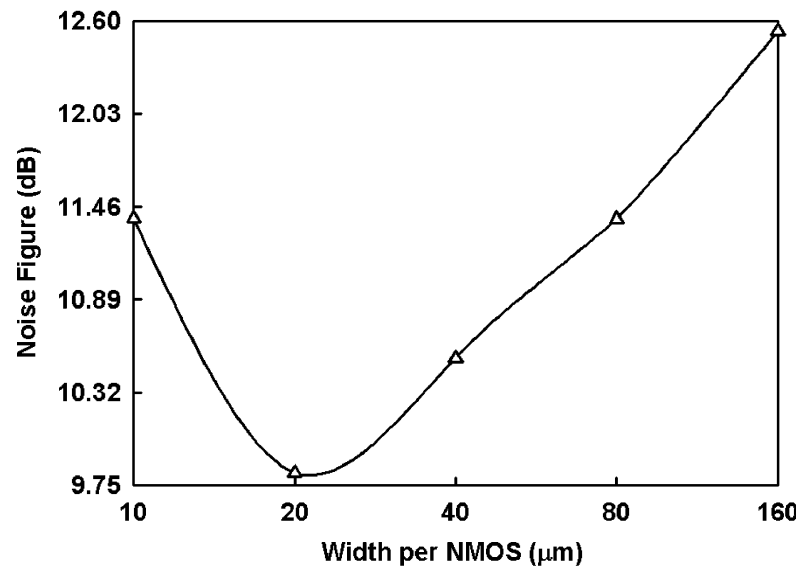

(b)

Fig. 13. Theoretical predictions of noise performances for the proposed BPFs with different transistor widths. (a) $g_{m}$ and $\gamma$. (b) NFs of the BPFs.

Fig. 13(a) and (b) plots the statistical results from which the following observations can be drawn. The value of $\gamma$, denoted by the curve with square symbols in Fig. 13(a), is highest at 1.63 with the smallest transistor width of $10.0 \mu \mathrm{m}$. However, $\gamma$ can be reduced and kept with a constant value of 1.10 when the width is larger than $80 \mu \mathrm{m}$. Conversely, $g_{m}$ is increased from 5.52 to $11.12 \mathrm{mS}$, corresponding to the increase of transistor 
width from 10 to $160 \mu \mathrm{m}$. Since the equivalent small-signal resistor between the drain and source terminals increases due to the nonquasi-static (NQS) effect [23], the higher value of $g_{m}$ is required to reduce the ohmic loss from the CCS TLs and the resistive loss in the channels of transistors. As revealed in (2), the noise current of the transistor is proportional to the product of $g_{m}$ and $\gamma$. Consequently, the NF, denoted by the curve with triangular symbols in Fig. 13(b), is $11.38 \mathrm{~dB}$, corresponding to the transistor width of $10 \mu \mathrm{m}$. Increasing the width of the transistor from 10 to $20 \mu \mathrm{m}$ causes the NF linearly reduced to its minimum value of $9.82 \mathrm{~dB}$. However, using the transistor width larger than $20 \mu \mathrm{m}$ in the BPF increases the resultant NF. These observations demonstrate the design tradeoff for minimizing the $\mathrm{NF}$ of the proposed BPF.

\section{CONCLUSION}

A TL-based active BPF was realized by using standard $0.18-\mu \mathrm{m}$ CMOS $1 \mathrm{P} 6 \mathrm{M}$ technology. The active BPF was constructed using the $Q$-enhanced half-wavelength resonators, which consisted of a differential nMOS cross-coupled pair and synthetic quasi-TEM CCS TL. In the CMOS process, the top metal (M6) and bottom metal (M1) formed the meandered traces and interconnections of the CCS TL and active devices. A mesh ground plane, located at M2, was sandwiched between M6 and M1, completing a vertical integrations for the proposed active BPF. The on-chip guiding characteristics of the CCS $\mathrm{TL}$ were theoretically extracted as the basic parameters for designing the $Q$-enhanced resonator. The circuit behaviors of the $Q$-enhanced resonator were theoretically investigated by conducting the differential- and common-mode analyses. The $Q$-enhanced resonator acted as a stable parallel resonator with $Q$-enhancement when the nMOS cross-coupled pair provided a negative resistance with an absolute value larger than that of a parallel resonator at the resonant frequency. The theoretical results indicate that the $Q$ factor of the resonator was elevated from 3.4 to 84 at $6.53 \mathrm{GHz}$. This resonator was applied in designing a prototype of a second-order BPF at $C$-band with a chip area of $1230 \mu \mathrm{m} \times 880 \mu \mathrm{m}$. The comparisons between the on-wafer measurements and simulations reveal that the proposed active TL-based BPF is feasible. Drawing $3.0 \mathrm{~mA}$ from a $1.8-\mathrm{V}$ supply, the prototype achieved $2.2-\mathrm{dB}$ insertion loss and 7.64-dB return loss when operating with a $1.14-\mathrm{GHz}$ BW at $6.02 \mathrm{GHz}$. The suppressions of spurious responses were 24 and $16.67 \mathrm{~dB}$ at 12.0 and $15.25 \mathrm{GHz}$, respectively. The high- and low-side rejections were 18.33 and $28.18 \mathrm{~dB}$ at 8.0 and $4.0 \mathrm{GHz}$, respectively. The input 1-dB compression point $\left(P_{1 \mathrm{~dB}}\right)$ and third-order intermodulation intercept point $\left(\mathrm{IIP}_{3}\right)$ were $-15.2 \mathrm{dBm}$ and $-9.6 \mathrm{dBm}$, respectively.

Additionally, a noisy two-port network was constructed to examine the NF of the proposed BPF. In the network, the total noise of the BPF consists of its transmission loss and the noise current produced by the nMOS cross-coupled pairs. The results of the analysis were compared with those of the simulations and experiments, revealing a difference among them of less than $7.2 \%$. Such agreements validate the feasibility of the proposed noisy network. Furthermore, the NF of the active TL-based BPFs designed with different transistor widths were theoretical analyzed using the proposed noisy network. A design curve demonstrating the tradeoff on minimizing the NF of the proposed active BPF was also reported.

\section{REFERENCES}

[1] D. Li and Y. Tsividis, "Design techniques for automatically tuned integrated gigahertz-range active $L C$ filters," IEEE J. Solid-State Circuits, vol. 37, no. 8, pp. 967-977, Aug. 2002.

[2] T. Soorapanth and S. S. Wong, "A 0-dB IL $2140 \pm 30 \mathrm{MHz}$ bandpass filter utilizing $Q$-enhanced spiral inductors in standard CMOS," IEEE J. Solid-State Circuits, vol. 37, no. 5, pp. 579-586, May 2002.

[3] X. He and W. B. Kuhn, "A 2.5-GHz low-power, high dynamic range, self-tuned $Q$-enhanced $L C$ filter in SOI," IEEE J. Solid-State Circuits, vol. 40, no. 8, pp. 1618-1628, Aug. 2005.

[4] C. H. Doan, S. Emami, A. M. Niknejad, and R. W. Brodersen, "Design of CMOS for $60 \mathrm{GHz}$ applications," in IEEE Int. Solid-State Circuits Conf., San Francisco, CA, Feb. 2004, pp. 440-441.

[5] H.-T. Tso and C.-N. Kuo, "40 GHz miniature bandpass filter design in standard CMOS process," in Silicon Monolithic Integr. Circuits RF Syst. Top. Meeting, Atlanta, GA, Sep. 2004, pp. 239-242.

[6] A. N. Mohieldin, E. Sanchez-Sinencio, and J. Silva-Martinez, "A 2.7-V 1.8-GHz fourth-order tunable $L C$ bandpass filter based on emulation of magnetically coupled resonators," IEEE J. Solid-State Circuits, vol. 38, no. 7, pp. 1172-1181, Jul. 2003

[7] S. Bantas and Y. Koutsoyannopoulos, "CMOS active- $L C$ bandpass filters with coupled-inductor $Q$-enhancement and center frequency tuning," IEEE Trans. Circuits Syst. II, Analog Digit. Signal Process., vol. 51, no. 2, pp. 69-76, Feb. 2004.

[8] Y.-C. Wu and M. F. Chang, "On-chip RF spiral inductors and bandpass filters using active magnetic energy recovery," in Proc. IEEE Custom Integr. Circuits Conf., Orlando, FL, May 2002, pp. 275-278.

[9] F. Dulger, E. Sanchez-Sinencio, and J. Silva-Martinez, "A 1.3-V 5-mW fully integrated tunable bandpass filter at $2.1 \mathrm{GHz}$ in $0.35 \mu \mathrm{m}$ CMOS," IEEE J. Solid-State Circuits, vol. 38, no. 6, pp. 918-928, Jun. 2003.

[10] C.-K. C. Tzuang, H.-H. Wu, H.-S. Wu, and J. Chen, "A CMOS miniaturized C-band active bandpass filter," in IEEE MTT-S Int. Microw. Symp. Dig., San Francisco, CA, Jun. 2006, pp. 772-775.

[11] K. Entesari, T. Vaha-Heikkila, and G. M. Rebeiz, "Miniaturized differential filters for $\mathrm{C}$ - and $\mathrm{Ku}$-band applications," in Proc. Eur. Microw. Conf., Munich, Germany, 2003, pp. 227-230.

[12] C.-C. Chen and C.-K. C. Tzuang, "Synthetic quasi-TEM meandered transmission lines for compacted microwave integrated circuits," IEEE Trans. Microw. Theory Tech., vol. 52, no. 6, pp. 1637-1647, Jun. 2004.

[13] H.-S. Wu, H.-J. Yang, C.-J. Peng, and C.-K. Tzuang, "Miniaturized microwave passive filter incorporating multilayer synthetic quasi-TEM transmission line," IEEE Trans. Microw. Theory Tech., vol. 53, no. 9, pp. 2317-2720, Sep. 2005

[14] H.-H. Wu, H.-S. Wu, and C.-K. C. Tzuang, "Synthesized highimpedance CMOS thin-film transmission line," in Silicon Monolithic Integr. Circuits RF Syst. Top. Meeting, Atlanta, GA, 2004, pp. 302-304

[15] R. Behzad, RF Microelectronics. Upper Saddle River, NJ: PrenticeHall, 1998, p. 228

[16] G. L. Matthaei, L. Young, and E. M. T. Jones, Microwave Filters, Impedance Matching Networks, and Coupling Structures. Norwood, MA: Artech House, 1980, ch. 8.

[17] G. D. Vendelin, A. M. Pavio, and U. L. Rohde, Microwave Circuit Design using Linear and Nonlinear Techniques. New York: Wiley, 1990, p. 103.

[18] P. A. Layman and S. G. Chamberlain, "A compact thermal noise model for the investigation of soft error rates in MOS VLSI digital circuits," IEEE J. Solid-State Circuits, vol. 24, no. 2, pp. 79-89, Feb. 1989.

[19] E. Hegazi, H. Sjoland, and A. A. Abidi, "A filtering technique to lower LC oscillator phase noise," IEEE J. Solid-State Circuits, vol. 36, no. 2, pp. 1921-1929, Feb. 2001.

[20] D. K. Shaeffer and T. H. Lee, The Design and Implementation of Low-Power CMOS Radio Receivers. Norwell, MA: Kluwer, 1999, pp. 52-53.

[21] B. Razavi, Design of Analog CMOS Integrated Circuits. New York: McGraw-Hill, 2001, pp. 212-213.

[22] G. Gonzalez, Microwave Transistor Amplifiers: Analysis and Design, 2nd ed. Upper Saddle River, NJ: Prentice-Hall, 1997, ch. 4.

[23] Y. Cheng and C. Hu, MOSFET Modeling \& Bsim3 User's Guide. Norwell, MA: Kluwer, 1999, ch. 10 


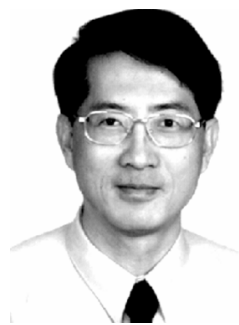

Ching-Kuang C. Tzuang (S'80-M'80-SM'92F'99) received the B.S. degree in electronic engineering from National Chiao Tung University, Hsinchu, Taiwan, R.O.C., in 1977, the M.S. degree from the University of California at Los Angeles (UCLA), in 1980, and the Ph.D. degree in electrical engineering from The University of Texas at Austin, in 1986.

From 1981 to 1984 , he was with TRW, Redondo Beach, CA, where he was involved with analog and digital MMICs. Since 1986, he has been with the Institute of Communication Engineering, National Chiao Tung University. In February 2004, he joined the Graduate Institute of Communication Engineering, Department of Electrical Engineering, National Taiwan University, Taipei, Taiwan, R.O.C., where he conducts research on advanced guiding structures for research and development of RF SOC, integrating active and passive microwave/millimeter-wave RF signal-processing components into a single chip. His research activities also involve the design and development of millimeter-wave and microwave active and passive circuits and the field theory analysis and design of various complex waveguide structures and large-array antennas. He has supervised 61 M.S. students and 21 Ph.D. students.

Dr. Tzuang helped in the formation of the IEEE Microwave Theory and Techniques Society (IEEE MTT-S) Taipei Chapter, and served as secretary, vice chairman, and chairman in 1988, 1989, and 1990, respectively. He recently served as the chairman of the Millimeter-Wave Industry Alliance, Taiwan Electrical and Electronic Manufacturers' Association (TEEMA), an assisted interest group in promoting standardization and application of millimeter-wave technology.

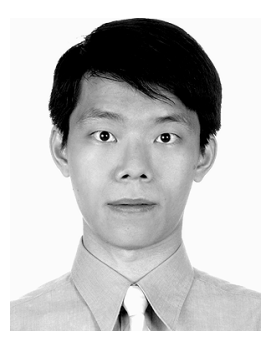

Hsien-Hung Wu (S'04) received the B.S. and M.S. degrees in electronic engineering from National Chiao Tung University, Hsinchu, Taiwan, R.O.C., in 1997 and 1999, and is currently working toward the Ph.D. degree in communication engineering at National Chiao Tung University.

His research interests include RF integrated circuits and computer-aided design (CAD) methodologies.

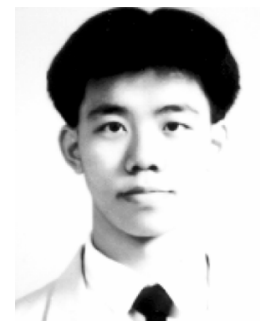

Hsien-Shun Wu (S'97-M'05) received the B.S. degree in electronic engineering from National Taipei University of Technology, Taipei, Taiwan, R.O.C. in 1999, and the M.S. and Ph.D. degrees in communication engineering from National Chiao Tung University, Hsinchu, Taiwan, R.O.C. in 2001, and 2005, respectively.

He is currently a Post-Doctoral Research Fellow with the Graduate Institute of Communication Engineering, National Taiwan University. His research interests include the design of wireless system modules and the development of synthetic waveguides for RF circuits.

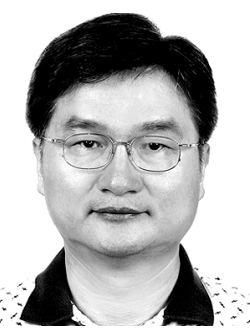

Johnsea Chen received the B.S. degree in materials science and engineering from National Tsing Hua University, Hsinchu, Taiwan, R.O.C., in 1977, and the M.S. and Ph.D. degrees in materials science from the University of Southern California, Los Angeles, in 1982 and 1985, respectively.

From 1985 to 1991, he was with Rockwell International, Science Center, Thousand Oaks, CA, where he was involved with II-VI compound semiconductors. In 1991, he cofounded the 4" GaAs Research and Manufacturing Corporation, Hsinchu, Taiwan, R.O.C. It was the first III-V compound semiconductor IC design and fabrication facility in Taiwan, R.O.C. In 1996, he joined Etron Technologies Inc., a silicon-based integrated circuit (IC) design company, as the Senior Executive involved with development and business-oriented operations. Early in 2002, he joined the Cadence Methodology Service Company (CMSC), Taipei, Taiwan, R.O.C., a wholly owned subsidiary of Cadence Design Systems, which is the world leading electronic design automation (EDA) developer and supplier. $\mathrm{He}$ helped to develop RF related materials and devices in Taiwan, R.O.C., over the past 15 years. His recent research interests focus on the development of RF SOC chips using advance CMOS-based silicon processes. 